\title{
LA DIMENSION PHILOSOPHIQUE DE LA THÉORIE DES CATASTROPHES
}

La théorie des catastrophes est issue des recherches du mathématicien français René Thom en Topologie et en Analyse différentielle sur la stabilité structurelle des applications différentiables'. Ses principes ont été exposés pour la première fois dans un article paru en 1966, intitulé "Une théorie dynamique de la morphogenèse " ${ }^{2}$. Elle est apparue sur la scène scientifique internationale six ans plus tard avec la publication, en 1972, du livre majeur de R. Thom, Stabilité structurelle et morphogenèse, écrit en 1968. La théorie se rattache mathématiquement aux travaux de Hassler Whitney sur les singularités des applications différentiables (1955) et à ceux de Poincaré et d'Andronov sur la théorie de la bifurcation des systèmes dynamiques ${ }^{3}$.

La théorie des catastrophes a connu un destin assez exceptionnel pour une théorie mathématique. Elle a eu très vite un grand retentissement dans la communauté scientifique internationale ${ }^{4}$. La théorie a été saluée, dès son apparition, comme une véritable révolution en mathématiques, comparable à l'invention du calcul différentiel et intégral au XVII siècle. Elle fournissait une méthode générale pour étudier les changements discontinus, les sauts qualitatifs. Elle a suscité de

1. Cf. le recueil d'articles de René Tном, Modèles mathématiques de la morphogenèse, Paris, Bourgois, 1980 (en abrégé : $M . M . M$.), p. 10. Une seconde source de la théorie a été, nous dit Thom, «la lecture des traités d'Embryologie, et notamment des livres de C. H. Waddington, dont les idées de " chréode" et de " paysage épigénétique " m'ont paru s'adapter très précisément au schéma abstrait que j'avais rencontré dans ma théorie de la stabilité structurelle des fonctions et applications différentiables " (ibid., p. 10). L'expression "théorie des catástrophes» est due à Christopher Zeeman. Thom n'avait parlé, quant à lui, dans son article inaugural de 1966, que de « points catastrophiques».

2. Reproduit dans $M . M . M$, p. $9-23$.

3. Sur les origines mathématiques de la théorie, cf. notamment Vladimir I. ARNOLD, Catastrophe Theory, Berlin/Heidelberg/New York/Tokyo, Springer-Verlag, 1984.

4. La presse elle-même lui a fait un large écho. 
grands espoirs chez les spécialistes de disciplines réputées non formalisables par les méthodes mathématiques traditionnelles (psychologie, éthologie, sociologie, etc.). La théorie allait faire entrer leur discipline dans le domaine envié de la rigueur. Des mathématiciens, dont Christopher Zeeman, de l'Université de Warwick en Angleterre, a été le chef de file, ont bâti des modèles "catastrophiques" pour rendre compte de phénomènes aussi divers que les révoltes dans les prisons, les krach boursiers, la propagation de l'influx nerveux, le traitement de l'anorexie mentale, etc. ${ }^{5}$. Emporté par son enthousiasme, C. Zeeman a même mis au point une "machine catastrophique", à base d'élastiques et de morceaux de carton, censée illustrer le fonctionnement de la théorie ${ }^{6}$.

Le succès de la théorie des catastrophes a peut-être été favorisé, à vrai dire, par la " magie " du mot catastrophe, qui avait incontestablement quelque chose de surprenant, et d'attirant dans un tel contexte. L'utilisation de ce mot a pu prêter à malentendu. Une catastrophe désigne dans le langage courant un événement tragique, un cataclysme, un bouleversement dramatique de l'ordre des choses. Or il n'y a rien de tel dans la théorie des catastrophes. Les catastrophes dont il y est question ont une signification mathématique bien précise. Il y a catastrophe, selon cette théorie, lorsqu'un changement continu dans les causes produit un changement discontinu dans les effets. En d'autres termes, la catastrophe est ce qui met en défaut l'adage causa aequat effectum. Elle est liée à l'idée centrale de discontinuité. Lorsqu'une fonction décrivant le comportement d'un système, par exemple, présente une discontinuité en un point, ce point est dit "catastrophique ". Si l'on avait gommé le côté sensationnel de la "théorie des catastrophes" en l'appelant autrement, il est probable que la théorie eût été moins remarquée. " La théorie de Thom, se demande par exemple J.-M. Lévy-Leblond, aurait-elle eu la même fortune eût-elle été baptisée : "théorie des transitions" ou " théorie des changements de forme "?" 7 . Il est à parier que non.

Après cette période d'engouement, la théorie a commencé à subir ses premières attaques, et même, à être sévèrement critiquée, "reflux nécessaire, dit Thom, d'une réception trop favorable ${ }^{8}$. Des mathé-

5. Cf. le recueil d'articles de Christopher Zeeman, Catastrophe Theory. Selected Papers, 1972-1977, Reading, MA, Addison-Wesley Publishing Company, Inc., Advanced Book Program, 1980.

6. Ibid., p. 8-23.

7. Jean-Marc LÊY-LeBlond, "Des mathématiques catastrophiques», Critique, 33, 1977, p. 439.

8. Stabilité structurelle et morphogenèse, Paris, Interéditions, 2e éd. 1977 (en abrégé : S.S.M.), préface, p. xi. 
maticiens "appliqués ", comme Hector J. Sussmann et Raphael S. Zahler, aux Etats-Unis, ont fait valoir que "les prétentions en faveur de la théorie [étaient] grandement exagérées, et que ses performances, du moins dans les sciences biologiques et sociales [étaient] insignifiantes $"{ }^{9}$. D'autres mathématiciens, comme S. Smale, médaillé Fields comme Thom, ont critiqué les fondements mathématiques de la théorie :

" [La théorie des catastrophes], dit Smale, rassemble deux des idées les plus fondamentales des mathématiques modernes : l'étude des systèmes dynamiques et l'étude des singularités des applications. Ces deux idées recouvrent, ensemble, un domaine très vaste, mais la théorie des catastrophes les réunit d'une manière arbitraire et forcée ${ }^{10}$.

Des physiciens ont joint leur voix au concert des critiques et ont manifesté un certain scepticisme quant à l'utilité pratique de la théorie. C'est le cas de J.-M. Lévy-Leblond, par exemple, qui se demande si les applications de la théorie des catastrophes à la biologie, à la linguistique, à l'éthologie, etc., ne seraient pas que "gadget conceptuel, placage analogique et alibi mathématique de disciplines en quête de respectabilité " 11 .

La polémique entre " catastrophistes " et détracteurs de la théorie semble s'être, depuis, quelque peu estompée. Les limites d'applicabilité de la théorie sont aujourd'hui mieux connues. Une sorte de consensus s'est établi au sein de la communauté scientifique pour accepter les applications "rigoureuses" de la théorie dans les domaines permettant l'élaboration de modèles quantitatifs précis, comme en Physique ou en Mécanique par exemple. En revanche, les avis restent partagés sur l'intérêt des modèles catastrophiques dans les domaines où la quantification est impossible (sociologie, etc.). Délaissant et dépassant cette controverse soulevée par les scientifiques concernant les limites d'applicabilité de la théorie des catastrophes, nous voudrions nous interroger ici sur ses implications épistémologiques et philosophiques. La théorie des catastrophes n'est pas, en effet, une théorie scientifique ordinaire. Ce n'est pas une théorie expérimentale comme le sont, par exemple, la théorie de la gravitation de Newton, la théorie de l'électromagnétisme de Maxwell ou la théorie de la relativité générale d'Einstein. Ce n'est pas non plus une théorie mathé-

9. Cf. revue Nature du 27 octobre 1977, cité par A. Woodcock, M. DAvis, in La Théorie des catastrophes, Lausanne, L'Age d'homme, 1984, p. 73.

10. Ibid., p. 75.

11. Art. cit. supra n. 7, p. 437. 
matique stricto sensu, c'est-à-dire une théorie de la Mathématique. Elle utilise certes les mathématiques (par exemple : la théorie des singularités des applications différentiables, la théorie de la bifurcation des systèmes dynamiques, certains théorèmes sur la structure des attracteurs en Dynamique qualitative, etc.), mais elle ne fait pas pour autant partie des mathématiques, car elle prétend fondamentalement parler du monde, de la nature. Nous voudrions montrer ici que le caractère atopique et atypique de la théorie des catastrophes, que certains pourraient être tentés de considérer comme une marque de non-scientificité, est en réalité le signe d'une rupture avec le paradigme qui gouverne la science contemporaine elle-même. Cette rupture est en même temps un retour, retour à une époque où science et philosophie n'étaient pas encore deux termes antithétiques, comme ils le sont devenus de nos jours. Thom réactive une vieille idée de la science, et peut-être la seule qui soit au fond tenable, la science en tant qu'elle permet de comprendre le réel, et pas seulement d'agir sur lui. Paraphrasant une formule célèbre, on pourrait caractériser la démarche de Thom en disant : "Jusqu'ici les savants n'ont fait que transformer diversement le monde; il s'agit maintenant de l'ipterpréter ${ }^{12}$.

\section{I. - LE formalisme MATHÉMATIQUe DE LA THÉORIE DES CATASTROPHES}

" Le spectacle de l'univers, écrit Thom au début de Stabilité structurelle et morphogenèse, est un mouvement incessant de naissance, de développement, de destruction de formes. L'objet de toute science est de prévoir cette évolution des formes et si possible de l'expliquer ${ }^{13}$.

Ce texte fixe le programme de la théorie des catastrophes : fournir des modèles mathématiques permettant de rendre compte non seulement de la stabilité des formes, mais aussi de leur évolution, de leur apparition et de leur disparition. La théorie des catastrophes peut être considérée comme une théorie générale de la morphogenèse, et il faut

12. Ibid., p. 438. Après la controverse de la fin des années 1970, la théorie des catastrophes connait aujourd'hui un nouvel essor. Les idées de René Thom ont été approfondies dans certains milieux, comme en sémio-linguistique avec W. Wildgen et J. Petitot ou en biologie avec $P$. Delattre et $Y$. Bouligand. Le contenu néo-aristotélicien de la théorie a été développé, à travers, notamment, les derniers travaux de René Thom sur les prégnances. Enfin, la théorie des catastrophes a donné lieu, depuis 1980, à d'importants développements philosophiques, surtout grâce à Jean Largeault, K. Pomian et J. Petitot.

13. S.S.M., p. 1 . 
entendre par morphogenèse tout processus créateur (ou destructeur) de formes. Une forme, telle que Thom la conçoit, se déploie sur un fond, sur un espace substrat dont l'apparence phénoménologique varie lorsqu'on le parcourt. "Le propre de toute forme, dit Thom, ... est de s'exprimer par une discontinuité des propriétés du milieu " ${ }^{14}$. Dans l'espace support de la morphologie (ordinairement l'espacetemps, mais ce peut être un espace plus complexe, comme en sociologie ou en éthologie), Thom distingue deux types de points : les points dits réguliers, qui correspondent aux zones de continuité du processus morphogénétique, et les points dits catastrophiques. Les points catastrophiques sont les seuls significatifs du point de vue morphologique. Au voisinage de chacun de ces points, l'apparence phénoménologique de l'espace substrat change, le processus morphogénétique présente une discontinuité : il y a création d'une forme. Avec le couple point régulier/point catastrophique, qui recoupe d'une certaine façon la distinction gestaltiste du fond et de la forme, Thom propose une méthode générale permettant d'analyser toutes les morphologies de l'expérience.

Pour expliquer une forme, on recourt d'ordinaire aux forces internes ou externes dont on suppose qu'elles en sont à l'origine. Toutefois, l'explication d'une forme par les forces se révèle souvent impossible car il arrive fréquemment que les forces agissantes ou bien sont inconnues (en biologie par exemple), ou bien sont d'une complexité telle qu'elles échappent à l'analyse et au calcul (érosion d'une falaise, par exemple). Le grand mérite de la théorie des catastrophes est qu'elle permet d'accéder à une " certaine compréhension des processus morphogénétiques, dit Thom, ... sans avoir recours aux propriétés spéciales du substrat des formes, ou à la nature des forces agissantes $"{ }^{15}$. Au lieu de déduire les formes de forces sous-jacentes, Thom projette au-dessus de l'espace substrat de la morphologie ce qu'il appelle un champ de dynamiques locales. À tout point $x$ du substrat, il associe un système dynamique, dépendant de $x$, qui va permettre de rendre compte des propriétés qualitatives du substrat en ce point. Un système dynamique requiert la donnée de deux éléments : un espace (une variété), noté $M$, support de la dynamique, et un champ de vecteurs, noté $X$, défini sur cet espace. Dans la théorie des catastrophes, la dynamique à l'origine de la morphologie ne joue donc pas dans l'espace substrat lui-même, mais dans un espace interne qui paramétrise les propriétés du substrat en chacun de ses points. Zee-

14. S.S.M., p. 9.

15. M.M.M., p. 10 . 
man appelle l'espace interne $\mathbf{M}$ " espace de comportement (behaviour state)" ou " espace des états (space state)", et l'espace support de la morphologie " espace de contrôle ".

La théorie générale des systèmes dynamiques est encore loin d'être achevée. C'est pourquoi $R$. Thom se borne, dans un premier temps (le seul que nous envisagerons ici), à l'étude des modèles n'utilisant que des systèmes dynamiques particulièrement simples: les systèmes dynamiques gradients pour lesquels le champ de vecteurs dérive d'une fonction potentiel $V$, et peut s'écrire $\vec{X}_{x}=-\overrightarrow{\text { grad }} V_{x}$. Thom suppose qu'en chaque point du substrat, la dynamique associée a atteint un point d'équilibre. Cela se traduit en termes mathématiques en disant que le point $c(x)$ qui paramétrise l'état du substrat en $x$ dans l'espace des états $M$ correspond à un minimum local du potentiel $V_{x}$ (puits de potentiel). Si le minimum local $c(x)$ du potentiel $V_{x}$ est stable, $x$ sera un point régulier du substrat. Cela signifie qu'au voisinage de $x$, l'état du substrat varie continuement autour de la valeur $c(x)$. En revanche, si le minimum local $c(x)$ du potentiel $V_{x}$ n'est pas structurellement stable, ce qui se produit lorsque la fonction $\mathrm{V}_{\mathrm{x}}$ change de type topologique au voisinage de $c(x)$ sous l'effet de petites perturbations, le point $x$ sera un point catastrophique. Dans ce cas, le minimum local c est détruit au voisinage de $x$, le plus souvent par collision avec un maximum local de $V_{x}$. Le point $c(x)$ cesse d'être un " attracteur " (c'est-à-dire un point d'équilibre) de la dynamique interne. En $x$, l'état du substrat saute brusquement de $c$ à $c_{1}$, où $c_{1}$ désigne un autre attracteur de la dynamique tel que $\mathrm{V}_{\mathrm{x}}\left(\mathrm{c}_{1}\right)<\mathrm{V}_{\mathrm{x}}(\mathrm{c})$. On dit que l'attracteur $\mathrm{c}_{1}$ a capturé l'attracteur c. À l'origine de toute catastrophe, il y a donc une situation d'instabilité : un attracteur de la dynamique bifurque. Les attracteurs instables correspondent aux points critiques dégénérés des potentiels. Thom découvre, et c'est là le résultat décisif, qu'un petit nombre seulement de singularités dégénérées (sept exactement) peuvent être à l'origine de morphologies structurellement stables dans l'espace-temps usuel $\left(R^{4}\right)$. Chacune de ces morphologies correspond à ce que Thom appelle une catastrophe élémentaire. Les sept catastrophes élémentaires pouvant se produire dans $R^{4}$ ont été baptisées, par ordre de complexité croissante: pli, fronce, queue d'aronde, papillon, ombilic hyperbolique, ombilic elliptique, ombilic parabolique. Ces noms sont dus à la forme que prend, dans chaque cas, la variété des équilibres, c'est-à-dire le lieu des minima des potentiels dans l'espace produit $\mathbf{R}^{4} \times \mathbf{R}^{n}$, où $\mathbf{R}^{n}$ désigne l'espace de comportement. Les morphologies dans l'espace substrat (les seules observables en pratique) sont obtenues par projection sur l'espace substrat de l'ensemble des points $S$ de la variété des équilibres où le plan tangent 
à la variété est "vertical ", c'est-à-dire "parallèle " à $R^{n 16}$. D'une façon générale, on peut montrer que le nombre de catastrophes élémentaires sur un espace donné dépend uniquement de la dimension de cet espace. La dimension de l'espace substrat borne la complexité topologique des catastrophes qui peuvent s'y déployer. La géométrie gouverne la topologie. Ces résultats requièrent pour leur démonstration des théorèmes profonds et difficiles, qu'il ne saurait être question d'exposer ici. Nous renvoyons pour cela aux ouvrages de Thom luimême, ou à celui, plus accessible, de Poston et Stewart ${ }^{17}$. Nous nous contenterons de présenter ici une des applications les plus connues de la théorie des catastrophes, qui, en dépit de sa simplicité, est tout à fait représentative de la méthode et des pouvoirs de la théorie. Il s'agit du modèle de l'agressivité du chien élaboré par C. Zeeman, fondé sur l'utilisation de la catastrophe de la fronce ${ }^{18}$.

Zeeman part des travaux de $\mathrm{K}$. Lorenz sur le comportement animal. K. Lorenz a montré, dans son livre sur l'agression ${ }^{19}$, que l'agressivité d'un chien dépendait de deux facteurs indépendants et antagonistes : la peur et la colère, corrélés à des expressions faciales caractéristiques. La peur et la colère sont des motivations conflictuelles qui induisent chacune un mouvement contraire à celui induit par l'autre. La peur provoque un mouvement de fuite et diminue la propension à l'agressivité, alors que la colère l'augmente. Lorsque l'une ou l'autre seulement de ces motivations est présente chez un animal, son comportement est assez facilement prévisible. Le problème se pose lorsque l'une et l'autre sont simultanément présentes. La coexistence de ces deux tensions contraires chez un chien provoque un conflit dont il est bien difficile de prévoir l'issue. Si la peur et la colère sont aussi importantes l'une que l'autre, par exemple, il est peu probable qu'elles s'annuleront et que le chien restera immobile. Il fuira ou attaquera beaucoup plus sûrement, mais il est pratiquement impossible de prévoir a priori quelle attitude prévaudra. $K$. Lorenz exprime cette imprévisibilité en termes d'équiprobabilité :

16. En d'autres termes, si on note $\pi$ la projection canonique $R^{4} \times R^{n} \rightarrow R^{4}$, et $\chi$ la restriction de $\pi$ à la variété des équilibres (notée $M_{f}$ ), l'ensemble des points catastrophiques est l'image par $\chi$ de l'ensemble des points de $\mathbf{M}_{\mathrm{f}}$ où l'application $\chi$ est singulière, c'est-àdire où sa dérivée $\mathrm{D} \chi$ est de rang inférieur à 4 (cf. Tim Poston, Ian STEwart, Catastrophe Theory and its Applications, London/San Francisco/Melbourne, Pitnam, 1978, p. 173).

17. Ibid.

18. Cf. op. cit. supra n. 5, p. 3-8, 12-13 et 67-68.

19. Konrad LORENZ, L'Agression : une histoire naturelle du mal, trad. de l'allemand par Vilma FrITSCH, Paris, Flammarion, 1977 ("Champs», 20). 
« [La mimique d']un chien, dit-il, [où] la colère et la peur se partagent à parts sensiblement égales ... doit être suivie, dans la moitié des cas, d'agression, et dans l'autre moitié, de fuite $"{ }^{20}$.

En d'autres termes, pour un mếme état de peur et de colère, deux comportements antagonistes sont a priori possibles. Le comportement du chien est donc, pour certaines valeurs de la peur et de la colère, dit Zeeman, bimodal.

Pour rendre compte de cette bimodalité, et des variations brusques du comportement de l'animal observées dans certains cas, Zeeman utilise la catastrophe de la fronce. Il choisit comme variables de contrôle dans l'espace support de la morphologie les deux facteurs antagonistes de la peur et de la colère, notés respectivement $x_{1}$ et $x_{2}$, et comme variable de comportement, l'agressivité du chien, notée c. Plus précisẻment, car on ne peut parler du comportement agressif d'un chien qu'en termes de probabilités, il interprète la variable $c$, non pas comme un degré d'agressivité, ce qui n'aurait guère de sens, mais comme la probabilité pour que le chien ait, dans une situation donnée, un comportement agressif. Lorsque c croît, la probabilité pour que le chien ait un comportement d'attaque croît ; si c décroît, la probabilité pour que le chien ait un comportement de fuite croît.

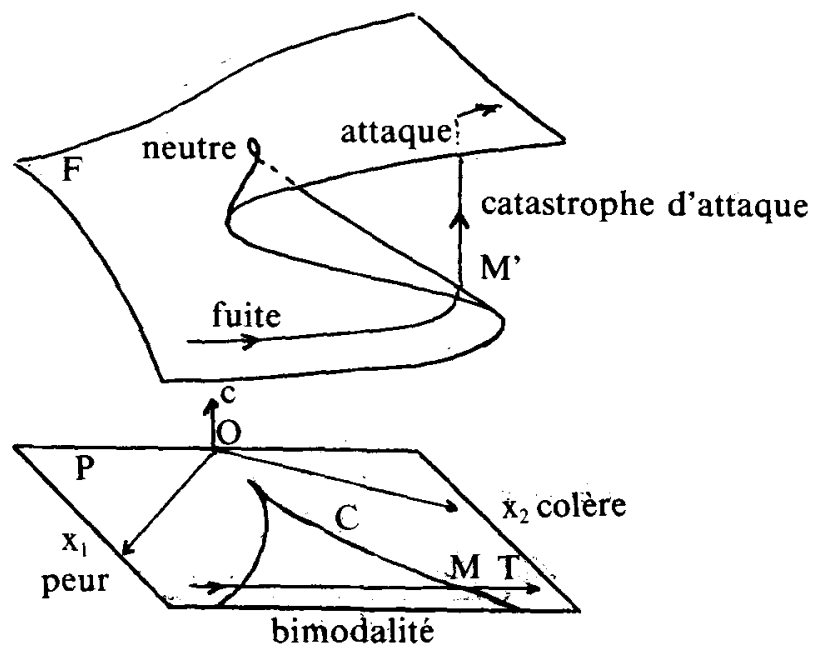

Fig. 1

20. Ibid., p. 100 . 
Lorsqu'on se déplace sur le plan de contrôle ( $P$ ), le comportement du chien se déplace sur la variété des équilibres (la fronce $F$ ). Au voisinage de l'origine, le comportement du chien est neutre. Lorsque la peur (respectivement la colère) domine, la probabilité pour que l'animal batte en retraite (respectivement attaque) est forte. À l'intérieur de la parabole semi-cubique $\mathrm{C}$, le comportement du chien est bimodal. Il y a catastrophe de fuite (ou d'attaque) lorsqu'on franchit, dans le plan de contrôle, l'une ou l'autre des branches de la parabole. Lorsqu'on suit le trajet $\mathrm{T}$, par exemple, qui correspond à une diminution continue de la peur doublée d'une augmentation continue de la colère, il viendra un moment où le chien finira, après avoir été dominé par une propension à la fuite, par changer brutalement de comportement et passera à l'attaque (en M) (cf. Fig. 1).

Le modèle de l'agressivité du chien qui, remarquons-le, est un modèle purement local puisqu'il ne formalise le comportement du chien qu'au voisinage de l'origine, a une valeur paradigmatique. La plupart des applications de la théorie des catastrophes se fondent, comme lui, sur la catastrophe de la fronce. Zeeman a formalisé à l'aide de ce type de catastrophe bon nombre de comportements bimodaux, comme ceux qu'on rencontre en sociologie (révolte dans les prisons), en physiologie (battements du cœur, transmission de l'influx nerveux), en économie, en psychiatrie (l'anorexie mentale), en embryologie (différenciation cellulaire), en physique (théorie des changements de phases, etc.). Quelques modèles catastrophiques se fondent certes sur des catastrophes plus complexes: Zeeman a recours par exemple à la catastrophe du papillon pour expliquer l'émergence de situations de compromis (en sociologie, par exemple). Thom donne des aperçus sur l'utilisation des ombilics pour étudier la morphologie du déferlement (ombilic hyperbolique pour formaliser le déferlement des vagues en hydrodynamique; ombilic elliptique en biologie pour expliquer la formation des organes pointus chez les êtres vivants : cil, flagelle, poil, piquants, etc.) ${ }^{21}$. Mais les modèles auxquels on aboutit, même s'ils sont parfois plus sophistiqués que celui de l'agressivité du chien, reposent fondamentalement sur les mêmes principes, et conduisent à des résultats qui sont, à bien des égards, comparables. C'est pourquoi nous n'entrerons pas davantage dans le détail des applications, mais nous allons nous tourner vers les principes que ces applications présupposent, c'est-à-dire vers les principes de base de la théorie des catastrophes, en essayant de dégager et

21. Cf. S.S.M., p. 75-81. 
de marquer leur originalité par rapport à ceux qui gouvernent la démarche scientifique contemporaine.

\section{II. - LES PRINCIPES ET L'ORIGINALITÉ DE LA THÉORIE DES CATASTROPHES}

L'originalité de la théorie des catastrophes tient d'abord, bien évidemment, à son objet. La théorie des catastrophes, en tant que théorie des formes, est une théorie des discontinuités. La science classique privilégie au contraire dans son analyse les phénomènes continus. Ce primat du continu, qui a pour corrélat un « mépris quasi permanent de la part des savants " pour la morphologie, notamment en physico-chimie, selon Thom ${ }^{22}$, a plusieurs raisons. Il tient d'abord à la difficulté même qu'il y a à mathématiser le discontinu. " Rien ne met plus mal à l'aise le mathématicien, dit Thom, qu'une discontinuité ${ }^{23}$. Il tient ensuite à la nécessité où se trouve le savant, afin d'être en mesure d'agir sur le monde, de pouvoir prédire l'évolution des phénomènes, c'est-à-dire de pouvoir déduire, connaissant l'état initial d'un processus, son état à un instant ultérieur quelconque. Une méthode classique pour prévoir l'évolution d'un phénomène consiste à " découper" le phénomène étudié en tranches temporelles infinitésimales, à déterminer la façon dont il se propage d'une tranche à la tranche suivante, et à intégrer ces évolutions locales en une évolution globale. La science dispose pour cela d'un outil remarquablement efficace, le calcul différentiel et intégral, développé au xviI siècle. L'utilisation de ces méthodes d'analyse différentielle conduit inévitablement à éliminer le discontinu du champ de l'investigation scientifique. Celui-ci est soit ignoré, soit ramené artificiellement à du continu. Il est à noter que la généralisation de ces méthodes d'analyse, dont l'efficacité n'est guère contestable, du moins à l'intérieur de certaines limites, a eu pour conséquence d'écarter, dans l'étude des phénomènes naturels, certaines branches des mathématiques, comme la géométrie ou la topologie, jugées peu performantes. C'est précisément sur ces disciplines (géométrie et topologie) que repose en partie la théorie des catastrophes.

Mais c'est surtout sur le plan de la méthode que la théorie des catas- 
trophes se démarque de la science contemporaine. La théorie des catastrophes, dit Thom, est

« une théorie herméneutique qui s'efforce, face à n'importe quelle donnée expérimentale, de construire l'objet mathématique le plus simple qui puisse l'engendrer ${ }^{24}$.

La théorie des catastrophes, en tant que théorie herméneutique, se développe comme une phénoménologie. Elle interprète le donné (c'est-à-dire les morphologies) phénoménologiquement, en le prenant tel qu'il apparaît, sans chercher à le déduire de processus élémentaires sous-jacents (forces physico-chimiques, par exemple). De ce point de vue, la théorie des catastrophes

" va à l'encontre, ajoute Thom, de la philosophie dominant actuellement, qui fait de l'analyse d'un système en ses ultimes constituants la démarche première à accomplir pour en révéler la nature. Il faut rejeter comme illusoire cette conception primitive et quasi cannibalistique de la connaissance, qui veut que connaître une chose exige préalablement qu'on la réduise en pièces $" 25$.

La " philosophie dominant actuellement " dont parle Thom est celle de la science contemporaine qui, pour comprendre une morphologie, cherche à la réduire, c'est-à-dire à l'expliquer à partir " d'entités plus petites, invariables et indestructibles, dont la combinatoire, l'interaction, va reconstituer la morphologie $"{ }^{26}$. L'explication scientifique traditionnelle est réductionniste. Le paradigme de l'explication réductionniste est l'explication atomistique, dont le principe est le suivant :

" positions et vitesses d'un système de $\mathrm{N}$ atomes, dit Thom, sont décrites par un point mobile dans un espace euclidien $R^{6 \mathrm{~N}}$ à $6 \mathrm{~N}$ dimensions $\left(\mathrm{x}_{\mathrm{i}}\right)$; les lois d'interaction entre ces particules permettent d'écrire un système d'équations différentielles : $\mathrm{dx}_{\mathrm{i}} / \mathrm{dt}=\mathrm{X}_{\mathrm{i}}\left(\mathrm{x}_{\mathrm{j}}\right)$ dont l'intégration donne l'évolution temporelle du système étudié ${ }^{27}$.

Malheureusement le nombre considérable d'équations à résoudre (songeons que pour une mole de gaz, $\mathrm{N}=6.10^{23}$ (nombre d'Avogadro), d'où un système avec $36.10^{23}$ équations) rend le programme

24. Paraboles et Catastrophes, Paris, Flammarion, 1983 (en abrégé : P.C.), p. 66.

25. S.S.M., p. 158.

26. "La Science malgré tout", in Encyclopaedia Universalis, Organum 1974, p. 8.

27. Ibid. 
réductionniste pratiquement ineffectuable. Seule une approche statistique permet d'avancer vers sa réalisation, et encore dans des cas tout à fait isolés et particulièrement simples. "Mais alors, fait remarquer Thom, il n'y a plus de morphologie $"{ }^{28}$. La forme se trouve dissoute au sein des phénomènes élémentaires à partir desquels on prétendait l'expliquer. La théorie des catastrophes, au rebours de la méthode réductionniste, ne décompose pas la forme en un ensemble d'entités atomiques, mais la prend au niveau d'organisation où elle apparaît. Elle sauvegarde l'autonomie du macroscopique et préserve ses voies d'intelligibilité. Thom qualifie l'approche propre à la théorie des catastrophes de structurale. Dans cette approche,

" on prend la morphologie $(\mathrm{X})$ en elle-même, à un certain niveau d'organisation, comme une combinatoire de champs morphogénétiques. On s'efforce de réduire l'arbitraire de la description du corpus en mettant en évidence ses régularités, ses symétries cachées, on se donne pour but d'engendrer en quelque sorte axiomatiquement la morphologie (X) à partir d'un petit nombre de formes susceptibles d'engendrer de nouvelles formes par agrégation spatiale ${ }^{29}$.

L'approche structurale ne cherche pas à atteindre, dans l'espace substrat de la morphologie, un niveau d'organisation élémentaire, mais introduit « au-dessus " de l'espace substrat un nouvel espace, de caractère mathématique et abstrait : l'espace de comportement. Plus précisément elle consiste à construire au-dessus de la morphologie un être géométrique que Thom appelle, en souvenir d'Héraclite, un $\log _{0}{ }^{30}$, c'est-à-dire, en théorie des catastrophes élémentaires, un potentiel organisateur susceptible de l'engendrer.

" La théorie des catastrophes, dit Thom, suppose [...] que les choses que nous voyons sont seulement des reflets et que pour arriver à l'être luimême, il faut multiplier l'espace substrat par un espace auxiliaire et définir dans cet espace produit, l'être le plus simple qui donne, par projection, son origine à la morphologie observée $»^{31}$.

28. Ibid., p. 9.

29. Modèles mathématiques de la morphogenèse, Paris, U.G.E., 1974 (Coll. 10/18, 887), p. 21-22.

30. "Si l'on veut bien admettre, écrit Thom, que le logos d'une chose est, chez Héraclite, cette structure formelle qui en assure l'unité et la stabilité, on conviendra que l'emploi assez particulier du mot forme dans cet ouvrage (c'est-à-dire S.S.M.) [en tant que classe d'équivalence d'une forme géométrique structurellement stable] en réalise une assez bonne approximation " (S.S.M., p. 323).

31. P.C., p. 85 
Ce doublement de l'espace morphologique concret, lieu de l'apparaître par un espace mathématique abstrait, lieu de l'être, rend incontestablement un son platonicien. Thom lui-même, du reste, compare les morphologies qui apparaissent dans l'espace substrat aux ombres de la caverne du livre VII de la République de Platon ${ }^{32}$. De la même manière que pour expliquer les ombres de la caverne, c'est-à-dire pour comprendre les « lois " qui gouvernent leur succession, il faut sortir de l'espace (le mur) sur lequel elles défilent, et se tourner vers l'extérieur, vers les choses réelles qui sont transportées au-dehors et vers le feu qui les éclaire, de la même manière, pour rendre compte des morphologies, dans la théorie des catastrophes, il faut sortir de l'espace substrat où elles apparaissent, et se tourner vers un être algébrico-géométrique qui les engendre. On peut parler, de ce point de vue, d'un platonisme de Thom : à l'origine des formes, simples reflets, il faut placer un monde idéel, un monde d'êtres mathématiques, celui des logoi, des potentiels organisateurs de la théorie des catastrophes élémentaires.

La méthode structurale caractéristique de la théorie des catastrophes n'est pas, bien sûr, incompatible avec la méthode réductionniste. Thom ne revendique, en effet, aucun exclusivisme de la méthode. Seulement l'avantage de la méthode structurale est qu'elle demeure opérante là où la méthode réductionniste est impraticable. C'est le cas notamment lorsque le nombre de paramètres qui gouvernent une morphologie est tel qu'il défie l'analyse quantitative ordinaire, comme dans ces phénomènes familiers que sont par exemple "les lézardes d'un vieux mur, la forme d'un nuage, la chute d'une feuille morte, l'écume d'un bock de bière $"{ }^{33}$. Une analyse quantitative des phénomènes de ce genre est ineffectuable : qui pourrait, en effet, prévoir avec précision la trajectoire que va suivre une feuille morte qui se détache d'un arbre? L'analyse quantitative doit ici céder la place à une approche moins ambitieuse, comme celle qu'autorise la théorie des catastrophes qui peut permettre d'expliquer ces formes en dévoilant les logoi qui les gouvernent et de rendre compte, non pas, bien sûr, de leurs caractéristiques quantitatives précises, mais de leurs déterminations qualitatives locales, et aussi de prévoir, dans une certaine mesure, leur évolution. La démarche de Thom peut être ici rapprochée de celle de Poincaré qui, dans un autre domaine, celui de la mécanique céleste, substituait à l'analyse quantitative du mouvement une analyse qualitative, lorsque la première ne pouvait aboutir. Ainsi,

32. Ibid.

33. S.S.M., p. 10 . 
dans le problème des trois corps (déterminer les positions, à un instant $t$ quelconque, de trois corps soumis aux seules forces d'attraction qu'ils exercent les uns sur les autres connaissant leurs positions et leurs vitesses à l'instant $\mathrm{O}$ ), Poincaré, après avoir montré qu'on ne pouvait calculer la solution, bien qu'elle existe et qu'elle soit unique, élabore une méthode qui permette de déterminer la forme des trajectoires et donne naissance à une nouvelle discipline : la Dynamique qualitative. La théorie des catastrophes peut être considérée d'une certaine façon comme l'héritière, au moins par sa méthode, de cette nouvelle discipline. Elle est en quelque sorte à la Physique, et à la science traditionnelle en général, ce qu'était, et ce qu'est encore, la Dynamique qualitative à la Dynamique classique.

La théorie des catastrophes est ensuite, et c'est ce qui contribue encore à la démarquer de la science contemporaine, une théorie " classificatoire ". Les idées de classe et de classification y jouent un rôle prépondérant, et cela en un double sens. Tout d'abord, la théorie vise à identifier, puis à classer toutes les singularités algébrico-géométriques qui peuvent donner naissance à des morphologies structurellement stables dans l'espace $R^{4}$ (au moins sous sa forme élémentaire). Thom établit "le catalogue de toutes les constructions possibles d'entités stables " ${ }^{34}$, de tous les logoi qui commandent la morphogenèse dans l'espace-temps. Ces structures algébriques constituent en quelque sorte l'a priori qui gouverne, au moins localement, toute morphologie empirique. La théorie des catastrophes est classificatoire en un second sens, à savoir eu égard à la manière dont l'explication s'y développe. Expliquer, dans la théorie des catastrophes, c'est rattacher une morphologie empirique à une structure archétypique. Connaître une morphologie, c'est reconnaître l'être algébrique qui est à son principe.

« Le modèle des catastrophes, dit Thom, est à la fois beaucoup moins, et beaucoup plus qu'une théorie scientifique; on doit le considérer comme un langage, une méthode qui permet de classifier, de systématiser les données empiriques, et qui offre à ces phénomènes un début d'explication qui les rende intelligibles ${ }^{35}$.

Cet idéal de classification évoque davantage, à vrai dire, les méthodes des taxinomistes du XvIII siècle (Linné, etc.) que celles de la science moderne. Pour Thom, comme pour les taxinomistes, la nature

34. M.M.M., p. 298.

35. "La théorie des catastrophes : état présent et perspectives", in op. cit. supra n. 5, p. 615 . 
a un sens, elle parle un langage qui lui est propre. Les choses ont une signification intrinsèque, contenue précisément dans leur logos, que l'analyse doit permettre de découvrir. Cette attitude choque le conventionalisme, qui est aujourd'hui la règle. Elle est inspirée par le désir de comprendre le réel, de le dévoiler dans son intelligibilité. La science moderne, quant à elle, ne se préoccupe guère de dévoiler le sens ni de saisir des essences, mais cherche avant tout à établir les lois permettant le calcul et la prédiction.

L'originalité de la théorie des catastrophes tient, enfin, sans parler du primat de la forme sur la force et du « retour de la géométrie " qui mériteraient à eux seuls de longs développements, à l'étendue proprement illimitée du domaine qu'elle est susceptible de recouvrir. La théorie des catastrophes a, en effet, une portée universelle. Elle peut s'appliquer à toutes sortes de phénomènes, appartenant à des secteurs très divers de la réalité. On peut la considérer comme une résurgence moderne du vieux rêve de la "mathesis universalis", de la Science universelle. Elle tranche de ce point de vue avec la spécialisation qui caractérise aujourd'hui le travail scientifique.

"Thom, dit à ce propos J. Petitot, se trouve être actuellement le centre d'un réseau de discours hétérogènes (de la tectonique des plaques à la dynamique des révolutions, de l'embryogenèse aux structures symboliques) que l'interdiscipline n'a jamais réussi à réaliser. Et c'est peut-être précisément là l'intérêt sociologique majeur de la théorie des catastrophes, de décloisonner les disciplines, de délocaliser la pensée, de reprendre des interrogations depuis longtemps désertées, de réarticuler des épaves de l'ontologie, divisée, brisée, morcelée par le développement techno-industriel ${ }^{36}$.

Le fondement théorique de l'universalité de la théorie des catastrophes est le principe de l'indépendance de la forme par rapport au substrat. D'après ce principe, la morphogenèse ne dépend pas de la nature des forces sous-jacentes, mais d'abord et avant tout de contraintes d'ordre géométrique et topologique. Une même forme peut apparaître sur des supports différents. La théorie des catastrophes est de ce point de vue une théorie de l'analogie. Elle pourrait même être entendue, selon Thom, comme la " première systématisation, assez générale de l'analogie $"{ }^{37}$ depuis Aristote. L'analogie, telle que Thom la conçoit, s'exprime en terme d'isomorphisme : deux mor-

36. "Catastrophes (théorie des) - un événement et un avènement ", in Encyclopaedia Universalis, Universalia 1978, p. 202.

37. P.C., p. 135. 
phologies empiriques sont analogues lorsqu'elles ont pour origine le même logos, le même être géométrique. La théorie des catastrophes établit des liens entre des phénomènes aussi éloignés que les transitions de phase, les révoltes dans les prisons, la différenciation cellulaire, etc. Elle fraye des passages " horizontaux " entre les disciplines scientifiques, entre la physique et la sociologie, entre la biologie et la linguistique, etc. Elle lutte contre l'éparpillement des savoirs en découvrant localement l'unité structurelle fondamentale de la nature.

\section{III. - L'ENJEU ÉPISTÉMOLOGIQUE ET LA DIMENSION PHILOSOPHIQUE DE LA THÉORIE DES CATASTROPHES}

Après avoir été accueillie très favorablement de manière quasi unanime par la communauté scientifique internationale, parfois par ceux-là mêmes qui allaient être par la suite ses plus farouchẹs détracteurs, la théorie des catastrophes s'est trouvée placée au centre d'une vive controverse. Différents types de critiques, touchant plusieurs de ses aspects, ont été formulés à son encontre. On lui a reproché notamment de ne pas être soumise au tribunal de l'expérience, et d'être de ce point de vue aussi peu scientifique que la magie ou que la psychanalyse ; de n'avoir qu'un pouvoir prédictif très limité ; d'être qualitative et non pas quantitative, locale et non pas globale. Toutes ces critiques convergent finalement vers le même thème : l'inefficacité pratique de la théorie. Les modèles catastrophiques ne permettent pas la prédiction ni, par conséquent, l'action efficace sur le réel. La théorie des catastrophes ne nous parle pas du monde. C'est une construction mathématique abstraite, coupée de la réalité, dont on ne peut même pas dire qu'elle est fausse puisqu'elle échappe au contrôle expérimental. C'est du point de vue du positivisme, une spéculation vide, et au fond, sans grand intérêt. Thom connaît, bien sûr, ces critiques. Il lui est même arrivé de s'en faire le porte-parole, et a pu, dans certains cas, contribuer à les articuler. Il ne les a pas éludées, mais y a au contraire longuement répondu. À travers ses réponses, qui excèdent le cadre étroit dans lequel la polémique s'est développée, se révèle ce qui nous paraît être la dimension véritable, l'ambition profonde de la théorie des catastrophes.

L'enjeu des réponses de Thom aux critiques formulées contre sa théorie dépasse, en effet, et de très loin, ce qui pourrait n'être après tout que la simple défense d'une théorie menacée. Elles débouchent 
sur une véritable mise en question, par une sorte de choc en retour, de la pensée scientifique contemporaine elle-même. Elles ébranlent les détracteurs de la théorie dans leurs convictions, et au-delà d'eux, toute une tradition scientifique. Elles interpellent l'idéologie positiviste et bousculent un certain nombre d'a priori, tout en donnant, et ce n'est pas là leur moindre intérêt, de nouveaux horizons à la recherche scientifique elle-même ${ }^{38}$.

Que sa théorie n'ait guère permis jusqu'ici d'étendre de façon décisive nos possibilités d'action sur le réel, Thom serait le premier à l'admettre.

« Il n'y a guère de doute, dit-il, que la critique portant sur l'inefficacité pragmatique des modèles de la théorie des catastrophes ne soit, pour l'essentiel, fondée. Mais il ne faut pas, ajoute-t-il, s'attendre à voir cette critique se développer beaucoup, parce que, si on la poursuivait, elle aboutirait à remettre en cause une bonne partie de la production scientifique contemporaine ${ }^{39}$.

On méconnaît, en effet, généralement ce que Thom appelle « la dégénérescence relativement rapide des possibilités de l'outil mathématique ${ }^{40}$ en science, dès qu'on s'éloigne du domaine où les mathématiques sont reines : la physique fondamentale et la mécanique. Lorsqu'on quitte ce domaine, où les mathématiques ne sont pas seulement, à vrai dire, un simple instrument, mais jouent un rôle constituant dans la détermination de l'objet scientifique lui-même, on entre dans le domaine de l'approximation. Les lois théoriques tirées de la physique pure ne permettent plus d'expliquer l'évolution temporelle des systèmes. Il faut leur adjoindre des hypothèses ad hoc, tirées généralement de considérations empiriques. La baisse de rendement de l'outil mathématique est déjà sensible en mécanique appliquée, et en dynamique des fluides. Elle s'accélère en Biologie, où les mathématiques ne servent guère qu'à façonner des modèles locaux qui n'ont qu'une portée pratique limitée. Elle est à son comble en éthologie, en

38. "Plus de théorie, et moins d'expérimentation », tel pourrait être le mot d'ordre que Thom donnerait à la science contemporaine. On pourra se reporter sur ce sujet à la communication de $R$. THOM à la séance de l'Académie des sciences du 19 novembre 1984 : « La méthode expérimentale : un mythe des épistémologues (et des savants?) », in La Philosophie des sciences aujourd'hui, sous la dir. de Jean HAMBURGER, Paris, GauthierVillars, 1986 ( Académie des sciences »), p. 7-20. "Une tradition - d'ailleurs douteuse-, dit Thom, disait, dans l'Antiquité, qu'il fallait "sauver les phénomènes » de l'appétit spéculatif incontrôlé des physiciens d'alors. À notre époque, c'est plutôt la pensée qu'il faudrait sauver de l'arrogante autorité de l'expérimentation " (ibid., p. 18).

39. M.M.M., p. 112 .

40. Ibid., p. 113. 
psychologie, et en sciences sociales, où le nombre de paramètres à prendre à compte est si élevé qu'il rend impossible ou illusoire toute tentative de modélisation quantitative précise. Les mathématiques ne servent plus guère alors qu'à fournir des "recettes statistiques, dit Thom, dont la légitimité même est suspecte $"{ }^{41}$. Cette baisse de rendement de l'outil mathématique en science reflète au fond une réalité " dont bien peu de scientifiques sont conscients, dit Thom, (et ce petit nombre préfère le couvrir du pieux voile de Noé), [à savoir] l'extrême misère théorique de la plupart des sciences $"{ }^{42}$. Nous touchons ici un thème central de l'analyse épistémologique de Thom : l'abandon par la science elle-même de sa tâche fondamentale de "théorisation " du réel. "La science, dit Thom, a oublié sa vocation première, celle qui fleurissait des présocratiques à Aristote, et qui était de nous faire comprendre la réalité ${ }^{43}$. Cette renonciation de la science à sa vocation théorique essentielle s'est produite à l'époque de Galilée et de Newton. Avec Galilée et Newton, on gagne bien en précision dans la description du mouvement des corps matériels, en éliminant le recours aux forces occultes, aux entéléchies, mais on perd de vue toute connaissance de la cause du mouvement. La science devient descriptive et cesse d'être explicative: elle donne le comment, mais non pas le pourquoi. Newton décrit mathématiquement, avec la loi de la gravitation en $k / r^{2}$ et la seconde loi de la dynamique $(\overrightarrow{\mathrm{F}}=\mathrm{m} \vec{\gamma})$, la manière dont se produisent les mouvements, mais n'en donne pas la raison. La loi de la gravitation est une relation fonctionnelle, qui permet le calcul et la prédiction, mais ce n'est pas une explication ultime de la réalité. La nature et l'origine véritables de la gravitation sont inconnues, et Newton ne formule du reste aucune hypothèse à son sujet :

« Jusqu'ici, dit-il, j’ai expliqué les phénomènes célestes et ceux des marées par la force de gravitation [...] Je n'ai pas encore pu parvenir à déduire des phénomènes la raison de ces propriétés de la gravité, et je ne façonne pas d'hypothèses (hypotheses non fingo) " ${ }^{44}$.

Newton découvre que la loi de la gravitation en $\mathrm{k} / \mathrm{r}^{2}$ lui permet de retrouver par le calcul les lois de Kepler et de rendre compte d'autres phénomènes, mais il avoue son impuissance à expliquer pourquoi deux masses s'attirent précisément selon cette loi, et exercent l'une sur

41. Ibid.

42. Art. cit. supra n. 26, p. 7.

43. Ibid., p. 6.

44. Isaac NEWTON, Principes mathématiques de la philosophie naturelle, reprod. de l'éd. de 1756, trad. du latin par la Marquise DU CHASTELLET modifiée, Paris, Blanchard, 1966, t. II, p. 178-179. 
l'autre une force inversement proportionnelle au carré de leur distance. On a bien cherché, à vrai dire, depuis Newton, à déduire la loi de la gravitation d'autres mécanismes, mais aucune de ces tentatives n'a abouti "sans prédire du même coup d'autres phénomènes qui n'existent pas $"{ }^{45}$. La gravité permet une bonne formalisation du mouvement, mais sa cause demeure mystérieuse, et elle l'est encore de nos jours, au point que l' " on admirerait à l'égal de Newton celui qui réussirait à expliquer la loi ${ }^{46}$. Dans le changement de paradigme galiléo-newtonien, on gagne incontestablement en efficacité, mais on perd en intelligibilité. La science tend à devenir un ensemble de recettes qui marchent, et qu'on applique sans trop savoir pourquoi elles marchent.
" $\mathrm{A}$ la fin du xvII siècle, dit Thom, on en [est] venu à décréter qu'après tout, il n'y avait aucune raison de chercher une explication quand on dis- posait d'une formule qui marchait bien. Et donc, la physique a adopté ce point de vue selon lequel les formules qui ont du succès doivent être pré- sentées dénuées d'explications. Les philosophes positivistes plus radicaux sont même allés jusqu'à soutenir que le devoir de la science était de four- nir un ensemble de recettes qui marchent bien et permettent ainsi des pré- dictions et une action efficace $"{ }^{47}$.

Reprenant à son compte l'hypotheses non fingo newtonien, la science se cantonne aujourd'hui, pour une bonne part, dans la recherche et dans la mise au point de procédés techniques permettant d'agir sur le réel et de prédire son évolution, sans se préoccuper outre mesure de leur assurer des fondements théoriques satisfaisants. De là, la prolifération des recherches expérimentales à laquelle on assiste actuellement, " sans autre ambition ni intérêt, dit Thom, que d'apporter une pierre minuscule et bien vite oubliée à l'édifice du savoir universel $"{ }^{48}$. L'analyse de Thom n'est pas sans rappeler ici, à vrai dire, celle développée, mais dans un autre contexte, par Heidegger pour qui, on le sait, la technique n'est pas non plus un avatar, un sous-produit de la science moderne, mais l'élément dans lequel elle se déploie et qui la détermine dans son essence même. Pour se convaincre de la

45. Richard Feynman, Mécanique I, Paris, Interéditions, 1979, p. 98.

46. Emile Borel, Le Hasard, Paris, Alcan, 1914, p. 3.

47. P.C., p. 33-34. «Une théorie physique, dit dans ce sens P. Duhem, n'est pas une explication. C'est un système de propositions mathématiques, déduites d'un petit nombre de principes, qui ont pour but de représenter aussi simplement, aussi complètement et aussi exactement que possible, un ensemble de lois expérimentales " (Pierre DuHEM, $L a$ Théorie physique. Son objet, sa structure, Paris, M. Rivière, 1914, p. 24).

48. Art. cit. supra n. 26, p. 7. 
convergence des vues du savant et du philosophe, il suffit de considérer ces trois propositions où Heidegger consigne les traits fondamentaux qui caractérisent la science à l'époque moderne :

"10 La science moderne, dit Heidegger, se fonde sur l'essence de la technique; $2^{\circ}$ l'essence même de la technique $n^{\prime} e s t$ rien de technique; $3^{\circ}$ l'essence de la technique n'est pas simplement l'œuvre de l'homme, qu'une supériorité et souveraineté humaines pourraient dompter par une attitude morale appropriée ${ }^{49}$.

Thom lui-même, du reste, se réfère à plusieurs reprises à Heidegger, et au jugement assez froid qu'il portait sur la science contemporaine :

" La science contemporaine, dit Thom, s'est lancée dans une vaste entreprise d'investigation généralisée (description) et, pour l'expérimentation, dans ce qui n'est guère qu'une forme active de bricolage. Ainsi s'est trouvée justifiée la condamnation glaçante de Heidegger en 1929 (quatre ans après la mécanique quantique, qui était déjà une superbe illustration du premier défaut): Die Wissenschaft denkt nicht (la science ne pense pas) $"{ }^{50}$.

La théorie des catastrophes pourrait-elle infirmer la « condamnation glaçante " de Heidegger et représenter le contre-exemple d'une tentative visant précisément à combler le fossé aujourd'hui grandissant qui sépare la science de la pensée ? Heidegger répondrait certainement que non, car la théorie des catastrophes, pas plus que la science en général, ne saisit l'être en tant qu'être, ni la différence ontologique. La réponse de Thom serait probablement différente, dans la mesure où la théorie des catastrophes retrouve, comme on va le voir, la dimension théorique, et même philosophique, qui a été perdue de vue par la science contemporaine, obnubilée par l'étendue de ses succès pratiques.

L'infirmité pratique, maintes fois soulignée, de la théorie des catastrophes est tout, en effet, sauf un signe de faiblesse, d'imperfection ou d'immaturité de la théorie. Thom, loin de la minimiser, la revendiquerait plutôt.

49. Qu'appelle-t-on penser?, Paris, P.U.F., 1973, p. 235.

50. "La science en crise ", Le débat, 18,1982, p. 39. $\dot{\hat{A}}$ notre connaissance, l'affirmation: «la science ne pense pas" ne se rencontre pas chez Heidegger avant 1951, en l'occurrence dans un cours du semestre de l'hiver 1951-1952, publié dans Was heißt Denken ? ( $Q u$ 'appelle-t-on penser?). Ce thème y est développé à plusieurs reprises. Nous lisons par exemple dans les premières pages : «... la science ne pense pas, et ne peut pas penser, et ceci à vrai dire pour son bonheur, c'est-à-dire pour préserver la démarche qui la définit en propre» (cf. op. cit. supra n. 49, p. 26). 
«Au fond, dit J. Largeault, [Thom] n'est pas fâché que sa théorie se prête mal aux souillures des applications quantitatives. Il met plus haut la gratuité, la spéculation et la compréhension qualitative des processus étudiés ${ }^{51}$.

La théorie des catastrophes a une autre ambition que la prédiction quantitative ou que l'action sur le réel. Sa visée n'est pas pratique ou pragmatique, mais théorique ou théorétique. Thom ne se propose pas simplement de décrire les morphologies qu'il étudie, mais cherche à les comprendre, à les expliquer, ce qui veut dire « substituer au visible compliqué de l'invisible simple ${ }^{52}$. La théorie des catastrophes explique les morphologies en construisant au-dessus de l'espace substrat où elles apparaissent, le logos, c'est-à-dire l'être algébrico-géométrique le plus simple permettant de les engendrer. Elle ne se propose pas de prédire ni de produire de nouvelles morphologies, mais de fonder les morphologies existantes dans l'être, c'est-à-dire de les déduire d'un être mathématique, logiquement et ontologiquement premier. Thom réhabilite ici un vieux thème, récusé par l'idéologie positiviste, selon lequel " toute connaissance est une connaissance par les causes ". La théorie des catastrophes explique les morphologies empiriques en remontant aux causes qui sont à leur origine, non pas bien sûr aux causes efficientes, qui jouent dans le même espace que la morphologie étudiée, mais aux causes formelles, qui jouent dans un autre espace (l'espace des états), et qui sont les seules vraiment rationnelles. Par l'exigence d'intelligibilité qui est la sienne, par sa volonté de ne pas s'en tenir au comment et de rechercher systématiquement le pourquoi des choses, par son souci de fonder l'apparaitre dans l'être, par sa prétention à l'universalité, la théorie des catastrophes possède une dimension philosophique essentielle. Thom lui-même la considère comme une "philosophie de la nature". Thom renoue ici avec une époque aujourd'hui révolue où science et philosophie n'étaient pas encore deux termes antithétiques, comme ils le sont maintenant devenus. Par son souci d'expliquer et de comprendre, et son refus de s'en tenir à "la recette qui marche", il se sent proche de penseurs comme Descartes ou comme Leibniz, plus proche en tout cas de Descartes que de Newton :

« Descartes, dit Thom, avec ses tourbillons, ses atomes crochus, etc., expliquait tout et ne calculait rien. Newton avec la loi de la gravitation en

51. Philosophie de la nature 1984, Université Paris XII - Val-de-Marne, 1984, p. 115, n. 25 .

52. Selon le mot du physicien J. Perrin, souvent cité par R. THOM (cf. par exemple in P.C., p. 83). 
$1 / r^{2}$, calculait tout et n'expliquait rien. L'histoire a donné raison à Newton et relégué les constructions cartésiennes au rang des imaginations gratuites et des souvenirs de musée. Certes le point de vue newtonien se justifie pleinement du point de vue de l'efficacité, des possibilités de prédiction, donc d'action sur les phénomènes ... [Mais] les esprits soucieux de compréhension, ajoute-t-il, n'auront jamais, à l'égard des théories qualitatives et descriptives, des présocratiques à Descartes, [cette] attitude méprisante du scientisme quantitatif ${ }^{53}$.

Par son goût pour le savoir pur, pour la spéculation gratuite, qui lui fait placer la contemplation plus haut que l'action, « audace énorme, dit J. Largeault, en un siècle où la recherche est cadastrée, soumise à des impératifs de rendement ou à des routines administratives ${ }^{54}$, Thom se sent aussi, et peut-être même surtout, proche des Grecs. De Platon, tout d'abord, dont la philosophie de la nature, dans le Timée, n'est pas sans évoquer, par le rôle qu'y joue la géométrie, la réduction thomienne des morphologies de l'espace-temps à un ensemble fini de structures algébrico-géométriques élémentaires. D'Aristote ensuite, ou plutôt de l'aristotélisme dont la théorie des catastrophes reprend, en les interprétant du reste très librement, un certain nombre de thèmes parmi lesquels l'hylémorphisme ${ }^{55}$, la distinction de l'acte et de la puissance $^{56}$, la théorie des catégories ${ }^{57}$, le concept d'analogie ${ }^{58}$, etc. Mais quels que soient les liens qui l'unissent à l'aristotélisme, les penseurs avec lesquels Thom se sent le plus d'affinité sont incontestablement les présocratiques, et notamment Héraclite, eux qui

« nous ont laissé, dit-il, des vues d'une si grandiose profondeur, [...probablement parce qu']à cette époque l'esprit était encore en contact quasi direct avec la réalité, les structures verbales et grammaticales ne [s'étant] pas interposées comme un écran déformant entre la pensée et le monde ${ }^{59}$.

53. S.S.M., p. 5-6.

54. Op. cit. supra n. 51, p. 143.

55. "L'attitude matérialiste, traditionnelle en Science, dit Thom, consiste à dire que l'existence précède l'essence ...; le modèle de la théorie des catastrophes va à l'encontre de cet axiome, car il présuppose que, dans une certaine mesure, l'existence est déterminée par l'essence, l'ensemble des qualités de l'être. On peut y voir une résurgence du schème aristotélicien de l'hylémorphisme : la matière aspirant à la forme» (M.M.M., p. 87).

56. Cf. P.C., p. 70.

57. Cf. M.M.M., p. 297.

58. Cf. P.C., p. 135.

59. «Avec l'arrivée des Sophistes, poursuit Thom, de la Géométrie euclidienne, de la Logique aristotélicienne, la pensée intuitive fait place à la pensée discursive, la vision directe à la technique de la preuve " (M.M.M., p. 167, n. 1). 
Thom lui-même rapproche à plusieurs reprises sa propre théorie, et la vision du monde qu'elle implique, des doctrines des présocratiques.

\begin{abstract}
« Nos modèles, dit-il par exemple, attribuent toute morphogenèse à un conflit, à une lutte entre deux ou plusieurs attracteurs; nous retrouvons ainsi les idées (vieilles de 2500 ans !) des premiers présocratiques, Anaximandre et Héraclite. On a taxé ces penseurs de confusionisme primitif, parce qu'ils utilisaient des vocables d'origine humaine ou sociale comme le conflit, l'injustice ... pour expliquer les apparences du monde physique. Bien à tort selon nous, car ils avaient eu cette intuition profondément juste : les situations dynamiques régissant l'évolution des phénomènes naturels sont fondamentalement les mêmes que celles qui régissent l'évolution de l'homme et des sociétés, ainsi l'usage des vocables anthropomorphiques en Physique est foncièrement justifié " 60 .
\end{abstract}

La théorie des catastrophes, qui considère toute forme comme le résultat d'un conflit entre attracteurs se partageant un même espace substrat, débouche sur une vision dialectique et héraclitéenne de l'univers. D'autres traits rapprochent Thom des présocratiques : d'abord l'inscription de l'homme dans la nature. Rien n'est moins thomien que l'anthropocentrisme ou le subjectivisme. Ensuite, le refus de toute transcendance. Les logoi archétypes ne sont pas des réalités transcendantes, à la manière des idées platoniciennes par exemple, mais sont internes aux morphologies qu'ils déterminent. La causalité des structures algébrico-géométriques s'exerce en restant immanente à l'espace substrat qu'elles informent. Un troisième trait rapproche Thom des présocratiques, c'est ce qu'on pourrait appeler son " antiglobalisme" . Thom récuse la possibilité d'un savoir humain qui réussirait à englober la totalité, à la façon du système hégélien, par exemple.

«S'il était effectivement possible, dit Thom, d'intégrer tous [les modèles] locaux en une immense synthèse, l'homme serait fondé à dire qu'il connaît la nature ultime de la réalité, puisqu'il n'existerait pas d'autre modèle plus global que celui-là ; je crois, personnellement, que c'est là une exorbitante prétention ; très vraisemblablement, l'ère des grandes synthèses cosmiques s'est définitivement close avec la relativité générale et il est bien douteux (et sans doute peu utile) qu'on tente de la rouvrir ${ }^{61}$.

Notre connaissance ne s'exerce que localement. Prétendre le contraire, et chercher à atteindre le réel dans son exhaustivité serait une forme de démesure. Les présocratiques ne pensaient pas autre-

60. S.S.M., p. 327.

61. Ibid., p. 7. 
ment, et notamment Héraclite, lui qui nous rappelle que la « nature aime à se cacher ${ }^{62}$, ce qui signifie que l'apparence, le non-être, est une composante essentielle de l'être, de telle sorte qu'on ne peut jamais dévoiler l'être dans sa globalité. Par l'exigence de localité qui est la sienne, Thom reprendrait certainement à son compte la sentence delphique qui en constitue une des plus belles et des plus célèbres expressions : «connais-toi toi-même ». Cette formule n'a rien à voir, en effet, avec une quelconque affirmation du pouvoir de la réflexion (en dépit de toutes les interprétations modernes allant dans ce sens qu'on a pu en donner), mais signifie d'abord et avant tout : connais tes limites, connais que tu es mortel, à l'intérieur d'un monde précaire et qui t'est en partie voilé. La connaissance n'a pas pour but de nous faire échapper à notre finitude, mais de nous la rappeler. Thom ne dit pas autre chose :

" On pourra peut-être démontrer, dit-il, [avec la théorie des catastrophes] le caractère inéluctable de certaines catastrophes, comme la maladie ou la mort. La connaissance ne sera plus nécessairement une promesse de réussite ou de survie ; elle pourra être tout aussi bien la certitude de notre échec, de notre fin ${ }^{63}$.

La théorie des catastrophes ne nous fait pas échapper à notre destin, mais " si elle est contemplative, dit J. Largeault, elle suffit à assurer le bonheur ${ }^{64}$.

Les références de Thom à la tradition philosophique occidentale, et plus particulièrement aux présocratiques, sont incontestablement séduisantes, même si elles peuvent paraître surprenantes dans le contexte mathématique de la théorie des catastrophes, où il est question de systèmes dynamiques, de singularités, d'attracteurs, etc. Elles tranchent, en tout cas, avec la

" hautaine indifférence, comme dit J.-M. Lévy-Leblond, voire [le] souverain mépris, comme l'affiche ouvertement tel ponte bourbachique, [avec lequel] la haute société mathématique considère les questions philosophiques - sans parler des querelles idéologiques. C'est que pour elle, ajoute

62. Fragment 123, in Hermann Diels, Die Fragmente der Vorsokratiker, Berlin, 6e éd., 1951. Cf. aussi Héraclite, Fragments, trad. du grec par Marcel Conche, Paris, P.U.F., 1986 (« Épiméthée »), p. 253.

63. Art. cit. supra n. 35 , p. 621.

64. Op. cit. supra n. 51 , p. 147. 
J.-M. Lévy-Leblond, les mathématiques constituent leur propre fin et sont devenues nécessaires et suffisantes à toute pensée digne de ce nom. Aux philosophes donc de venir y apprendre la langue définitive de la raison ... ${ }^{65}$.

Les références philosophiques de Thom contrastent avec cet « élitisme mathématique " et laissent entrevoir la possibilité d'un rapprochement entre la science et la philosophie. Mais elles soulèvent aussi un certain nombre d'interrogations, et d'abord celle, qui nous paraît fondamentale, de la pertinence d'une interprétation des doctrines philosophiques à la lumière d'une théorie mathématique, quand bien même s'agirait-il d'une théorie "qualitative " comme la théorie des catastrophes.

«Toutes les intuitions fondamentales en morphogenèse, dit Thom, se trouvent déjà dans Héraclite ; mon unique apport est de les avoir replacées dans un cadre géométrique et dynamique qui les rende un jour accessibles à l'analyse quantitative ${ }^{66}$.

Thom avoue ici sa dette, concernant les "intuitions fondamentales ", à l'égard de la doctrine d'un des premiers penseurs de la Grèce : la vision dialectique du monde qu'il propose est une géométrisation de celle qu'ébauchait Héraclite il y a plus de 2500 ans. Une telle géométrisation permet, selon lui, d'apporter de la précision aux " visions grandioses et profondes, mais un peu vagues, des premiers présocratiques $"{ }^{67}$. Elle doit même permettre de sauver les doctrines spéculatives de ces penseurs d'un déclin qu'on suppose inéluctable, en ôtant " le caractère irrémédiablement naïf et imprécis des images [qu'elles mettent] en jeu " ${ }^{68}$. L'intérêt proprement philosophique d'une telle géométrisation parait, il faut bien le reconnaître, assez mince. En effet, le caractère imprécis, que Thom déplore dans les notions philosophiques, tient en réalité à ce que ces notions sont fondamentalement surdéterminées : il y a toujours plus, dans les paroles des penseurs, que ce que nous y voyons de prime abord. Il paraît donc impossible de transcrire les doctrines philosophiques, sans les appauvrir et sans manquer leur sens véritable, en un langage mathématique qui, par définition même, en supprimerait l'épaisseur et la profondeur. Ce n'est peut-être pas un hasard, du reste, si le rêve leibnizien d'une

65. Art. cit. supra n. 7, p. 431.

66. S.S.M., p. 10.

67. Ibid., p. 6.

68. Ibid. 
" caractéristique universelle " (transcription algébrique des concepts, et formalisation du raisonnement), un moment caressé par Thom, n'a pu aboutir. En réalité, l'apport de la théorie des catastrophes n'est pas là ; il ne réside pas dans une quelconque géométrisation du sens, au demeurant hypothétique, ni dans un devenir-science de la philosophie, mais bien plutôt dans ce qui serait un devenir-philosophie de la science elle-même. La théorie des catastrophes montre la possibilité, et même la nécessité, d'une science moins pratique, et plus théorique, moins technique et plus philosophique. La démarche de Thom n'est pas sans rappeler ici, à vrai dire, celle suivie, de façon tout à fait indépendante et dans un tout autre domaine, par un autre penseur contemporain : celle de Heidegger lui-même. Heidegger dénonce, en effet, lui aussi l'emprise de la pensée technicienne et calculante sur la pensée contemporaine, lui aussi se réfère aux présocratiques (comme à des penseurs qui se mouvaient encore dans le domaine aujourd'hui enfoui de la vérité de l'être). Et de la même manière que Heidegger appelle de ses vœux la venue d'une pensée plus pensante, Thom, un peu de la même façon, plaide pour une science plus " scientifique ", c'est-à-dire plus philosophique, car " on ne voit pas, dit-il, ce que pourrait être une science, sinon la philosophie elle-même ${ }^{69}$. La théorie des catastrophes ouvre ici la voie. C'est une théorie scientifique, et en même temps une philosophie, non pas, bien sûr, au sens d'un corps de propositions doctrinales, mais en tant qu'elle possède une dimension philosophique fondamentale et place la contemplation plus haut que l'action, la théorie plus haut que la pratique. Seul l'avenir pourra nous dire si l'œuvre de Thom n'est que le rejeton tardif d'une époque à jamais révolue où la science et la philosophie faisaient bon ménage, ou si au contraire, comme nous voulons le croire, elle ouvre une ère nouvelle où la science, redevenue consciente de sa mission théorique fondamentale, ne se contentera plus de prédire et de calculer le réel, mais cherchera aussi à le comprendre.

Alain Boutot.

69. Art. cit. supra n. 26, p. 10. 